\title{
Die-to-Die Adhesive Bonding Procedure for Evanescently-Coupled Photonic Devices
}

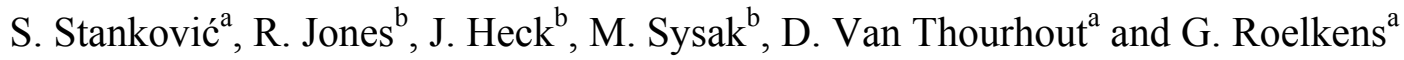 \\ ${ }^{a}$ Department of Information Technology, Ghent University - imec, Sint- \\ Pietersnieuwstraat 41, 9000 Gent, Belgium \\ ${ }^{\mathrm{b}}$ Intel Corporation, 2200 Mission College Blvd. SC12-326, Santa Clara, CA 95054, USA
}

\begin{abstract}
Recently demonstrated evanescent hybrid III-V/Si lasers are mostly based on molecular bonding of a III-V die on an SOI photonic wafer. This procedure requires ultra-clean and smooth bonding surfaces and might be difficult to implement in an industry-scale fabrication process. As an alternative, we present a die-to-die adhesive bonding procedure, using a DVS-BCB polymer. We achieved less than 100nm-thick bonding layers that enable evanescent coupling between III-V and silicon. The process shows good robustness and bonding strength, with a break-down shear stress of $2 \mathrm{MPa}$. The process can be scaled-up to a multiple die-to-wafer bonding procedure.
\end{abstract}




\section{Introduction}

Silicon photonics, based on the silicon-on-insulator (SOI) material platform, is considered as the technology of choice for the integration of photonic devices with microelectronic circuits. However, the fabrication of efficient light sources in silicon photonics is challenging due to silicon's indirect bandgap. Heterogeneous integration, achieved through the bonding of III-V semiconductor materials on a SOI platform, is the most promising approach to address this problem. Among several schemes to couple the light from the III-V active medium into the SOI waveguide (1), evanescent optical coupling is the most promising one and requires no additional coupling structures (2), although the active material and the silicon waveguide need to be within several hundred nanometers. Several evanescent hybrid III-V/Si lasers based on direct bonding were reported (2-5), but this technique is very sensitive to surface topography, contamination or presence of particles and may not be sufficiently robust for industrial-scale fabrication where such strict requirements are difficult to meet.

Compared to direct bonding, adhesive bonding is more tolerant to surface topography and particle contamination. It has been used for hybrid integration of photonic and electronic circuits (6). Both thermoplastic polymers, like SU-8 (7) and thermosetting polymers, such as polyimide and $\mathrm{BCB}(8)$, are used as adhesives. Our heterogeneous integration scheme assumes bonding unprocessed III-V dies on top of pre-patterned SOI waveguide circuits (see Figure 1a). Precise bonding alignment is not required since the III-V dies are processed after the bonding. Post-bonding thermal budget should allow $350{ }^{\circ} \mathrm{C}$ processing of the III-V components. Therefore, a thermosetting polymer, 
divinylsiloxane-bis-benzocyclobutene (DVS-BCB), also referred to as BCB, was chosen. It is a well-known material that is used both for wafer-to-wafer (8) and die-to-wafer bonding processes (9). Recently, the fabrication of evanescently-coupled photodetectors (10), hybrid III-V/Si lasers (11) and several other photonics devices (12) using BCB bonding has been demonstrated, but in these cases, a manual bonding procedure was used. This resulted in a difficult-to-control bonding procedure, which prevents scaling-up to an industrial-level fabrication. In this paper, we report a machine-based $\mathrm{BCB}$ bonding process providing thin bonding layers $(<100 \mathrm{~nm})$, suitable for the fabrication of evanescently-coupled photonic devices, specifically hybrid III-V/Si lasers.

\section{Experimental Setup and the Bonding Procedure}

We use $1.2 \mu \mathrm{m}$ wide silicon rib waveguides (see Figure 1b), surrounded by $3.4 \mu \mathrm{m}$ wide and $220 \mathrm{~nm}$ deep trenches. The silicon slab and the buried oxide layers are $500 \mathrm{~nm}$ and $1 \mu \mathrm{m}$ thick, respectively. We focus on a die-to-die bonding procedure that can eventually be scaled-up to a multiple die-to-wafer bonding where III-V dies would be bonded to a full SOI wafer. This is a cost-effective approach in heterogeneous integration since III-V material is usually required only on a small area of the SOI photonic wafer. The size of the III-V dies used in our experiments varies from $5 \mathrm{~mm} \times 5 \mathrm{~mm}$ to $8 \mathrm{~mm} \times 8$ $\mathrm{mm}$. The SOI dies are either $20 \mathrm{~mm} \times 20 \mathrm{~mm}$ or $10 \mathrm{~mm} \times 10 \mathrm{~mm}$ in size. In our tests, we use dry-etch Cyclotene 3022-35 which gives the thinnest BCB cured films $(1 \mu \mathrm{m}-2.4$ $\mu \mathrm{m})$ compared to other resins from the Cyclotene 3000 Series (13). To achieve bonding layers thinner than $1 \mu \mathrm{m}$, we dilute $\mathrm{BCB}$ with mesitylene in different volume ratios. 
We use Süss MicroTec ELAN CB6L wafer bonder, which can handle wafers, but not the small dies that are used in the experiments. Therefore, both the III-V and SOI dies are temporarily attached to $100 \mathrm{~mm}$ diameter Pyrex glass wafers acting as the die carriers. After attaching the dies, using a thermal release tape Revalpha No.31950E from Nitto Denko, we mount the carrier wafers on the transport fixture of the wafer bonder. The cross-section of this die-to-die bonding setup is given in Figure 2a. To compensate the thickness difference between the dies and the metallic spacers inserted between the wafers, additional silicon stubs are attached to the bottom carrier wafer (see Figure 2a). Prior to bonding, the metallic spacers that are the integral part of the transport fixture rest on these stubs, providing a gap between the SOI die and the III-V die. Once the metallic spacers are retracted, the III-V die is brought into contact with the SOI die (see Figure $2 b)$.

The bonding process starts with the cleaning of the dies. The SOI die cleaning is performed by dipping it into a Standard Clean 1 (SC-1) solution heated to $70{ }^{\circ} \mathrm{C}$, for 15 minutes. After this, adhesion promoter AP3000 is spin-coated on the SOI die, followed by spin-coating of the BCB:mesitylene solution. The SOI die is then baked for 4 minutes at $100{ }^{\circ} \mathrm{C}$, to let mesitylene evaporate and to stabilize the BCB film. Finally, the SOI die is mounted on a carrier wafer and placed on the transport fixture. Prior to bonding, the III-V die is attached to the carrier wafer and two sacrificial layers, an InP and InGaAs layer, are removed by selective wet etching, which also removes particles and contaminants from the III-V die surface. The III-V die is then rinsed with DI water, dried and mounted on the transport fixture, which is then loaded into the processing chamber. The bonding recipe starts with the evacuation of the processing chamber and heating it to 
$150{ }^{\circ} \mathrm{C}$. After 5 minutes in vacuum, the dies are brought into contact and kept at $150{ }^{\circ} \mathrm{C}$ for 20 minutes, while applying the pressure. The actual bonding area is limited by the III$\mathrm{V}$ die size and is $400-800$ times smaller than the area of the bonding head of our wafer bonder. Although the size of the III-V dies slightly varied in our tests, we kept the actual bonding pressure (the bond force per area of the III-V die) in the range of 4-5 bar (400$500 \mathrm{kPa}$ ). After keeping the dies pressed for 20 minutes at $150{ }^{\circ} \mathrm{C}$, the temperature is increased up to $240{ }^{\circ} \mathrm{C}$, with a ramp of $1.6{ }^{\circ} \mathrm{C} / \mathrm{min}$. This ramp allows a re-flow of $\mathrm{BCB}$ during the curing and is suggested by the $\mathrm{BCB}$ manufacturer (13). The bonding head is raised at $180{ }^{\circ} \mathrm{C}$ and no pressure is applied afterwards. This is done to avoid the additional pressure on the dies that would occur due to a "swelling" of the Revalpha tape at its release temperature of $200{ }^{\circ} \mathrm{C}$. Also, the nominal curing temperature of $250{ }^{\circ} \mathrm{C}(13)$ is reduced to $240{ }^{\circ} \mathrm{C}$ to minimize the residual adhesiveness of the Revalpha tape. Upon reaching $240{ }^{\circ} \mathrm{C}$, the dies are kept at this temperature for 1 hour in a $\mathrm{N}_{2}$ atmosphere. After the curing, the bonded samples are cooled down (at $\sim 6{ }^{\circ} \mathrm{C} / \mathrm{min}$ ) and unloaded from the processing chamber. The InP substrate on a III-V die is then grinded down to $\sim 70 \mu \mathrm{m}$, while the residual InP is removed by wet etching, leaving a thin III-V film with the functional layers bonded to the SOI die, ready for further processing.

\section{Experimental Results}

\section{Bonding Layer Thickness}

Initially, we tested the impact of the spin-coating rotation speed and the $\mathrm{BCB}$ volume ratio in $\mathrm{BCB}$ :mesitylene solutions on the thickness of the $\mathrm{BCB}$ layers. $\mathrm{BCB}$ solutions 
were spin-coated on flat InP dies and cured. The results showed that BCB content in the solution has more impact on the film thickness than the rotation speed. For a 1:3 BCB:mesitylene solution (25\% BCB volume content), average thicknesses achieved with $7000 \mathrm{rpm}$ and $3000 \mathrm{rpm}$ rotation speeds were $95 \mathrm{~nm}$ and $115 \mathrm{~nm}$, respectively, while for 2:3 BCB:mesitylene solution (40\% (v/v)) the respective values were $155 \mathrm{~nm}$ and $190 \mathrm{~nm}$. Other tests showed that spin-coating BCB on a patterned SOI die, at speeds above 5000 rpm, produced a non-uniform film immediately after spin-coating. To avoid this, we fixed our spin-coating speed at $3000 \mathrm{rpm}$ and used only the BCB:mesitylene solution ratio as a variable parameter to achieve the desired bonding layer thickness.

To measure this thickness, a cross-section is made through a bonded III-V film, using a focused ion beam (FIB) tool, and inspected using SEM. In the initial tests, using a 1:7 BCB:mesitylene solution $(12.5 \%(\mathrm{v} / \mathrm{v})), 15 \mathrm{~nm}$ thin bonding layers above the $\mathrm{Si}$ rib waveguide were achieved, but occasional peeling of the III-V film during wet etching and voids in the BCB film could be observed, indicating a too much diluted solution. Thus, a 1:3 BCB:mesitylene solution $(25 \%(\mathrm{v} / \mathrm{v}))$ was used and it gave an improved bonding yield and $\mathrm{BCB}$ layers of around $45 \mathrm{~nm}$, as shown in Figure 3a. After this, we started applying AP3000 adhesion promoter to the SOI die, prior to spin-coating BCB. Consequently, III-V film peeling was drastically reduced and high-quality III-V films were obtained, as shown in Figure 3b. In this case, the BCB layer thickness increased to $90 \mathrm{~nm}$, probably due to better BCB adhesion to the silicon surface and the increased viscosity during spin-coating. Using this bonding procedure and 25\% (v/v) BCB solution, we have performed 28 bonding tests. We considered a test successful if more than $80 \%$ of the original III-V film surface remained bonded to SOI die after the InP substrate removal. According to this criterion, 24 out of 28 tests ( $>85 \%)$ were successful. 


\section{Bonding Layer Uniformity}

Initially, several tests were performed in which pure InP dies were bonded on top of SOI waveguides. After the bonding, the InP was completely removed by wet etching, exposing the cured BCB film. On top of this film, a 40nm-thin layer of gold was deposited after which an optical profilometer (Wyko NT3300) was used to check the surface topography. A typical surface profile is shown in Figure 4. At the locations of the $220 \mathrm{~nm}$ deep trenches, surrounding the silicon rib waveguides (as shown in Figure 1b), 10-20 nm deep notches in the BCB surface were measured. This corresponds to a degree of planarization larger than $90 \%$, confirming the good planarization properties of BCB. In the bonding tests with the III-V dies with epitaxially grown layers, uniformity was assessed by measuring the BCB layer thickness at several locations, using a FIB tool. Results show that $\mathrm{BCB}$ is thicker at the die centre and thinner near the edges. The worst observed bonding layer thickness variation in a single die is from $20 \mathrm{~nm}$ to $120 \mathrm{~nm}$. By properly designing hybrid III-V/Si evanescent laser, such non-uniformity can be tolerated, and the confinement factor greater than $3 \%$ can be achieved in the III-V active region comprising 8 quantum wells (14). An optical profilometer was used to measure the III-V die surface topography prior to the bonding to correlate it to the bonding yield and the bonding layer uniformity. However, the measurements showed that there is no strong correlation between III-V die topography and the BCB bonding layer uniformity, nor the bonding yield. Another possibility is that the BCB layer non-uniformity is due to a nonuniform bonding pressure distribution and bending of the top Pyrex carrier wafer. As suggested in (15), a graphite foil between the III-V dies and the bonding head can be used to improve the bonding pressure uniformity. 


\section{$\underline{\text { Shear Stress Tests }}$}

Eleven bonded samples with standardized die sizes $(5 \mathrm{~mm} \times 5 \mathrm{~mm}$ for III-V dies and $10 \mathrm{~mm} \times 10 \mathrm{~mm}$ for SOI dies) were prepared using $25 \%(\mathrm{v} / \mathrm{v}) \mathrm{BCB}$ solution. The shear strength was measured using a Dage 4000 shear tester. The SOI die was clamped to the fixed stage while a lateral force was applied to the side of the III-V die. The bottom edge of the tool was $100 \mu \mathrm{m}$ above the bond interface and the shear speed was set to 700 $\mu \mathrm{m} / \mathrm{sec}$. The shear force, defined as the maximum force immediately before die breakage, was measured. The shear stress, calculated using the bonded area, ranged from $1.98 \mathrm{MPa}$ to 2.02 $\mathrm{MPa}$, with an average value of $2 \mathrm{MPa}$ and standard deviation $0.017 \mathrm{MPa}$. Since this is a destructive test, the BCB bonding layer thickness was measured in four control samples, additionally bonded using the same process parameters. The BCB thickness in the control samples varied between $32 \mathrm{~nm}$ and $83 \mathrm{~nm}$. Such a small variation (within 1\%) in the shear stress indicates there is no significant impact of the BCB thickness on the bond strength. It also confirms a very good robustness of our bonding process, while the average shear stress value of $2 \mathrm{MPa}$ suggests a solid bonding strength.

\section{Conclusions}

We developed a die-to-die DVS-BCB bonding procedure for the heterogeneous integration of III-V dies and SOI photonic waveguides. The demonstrated BCB bonding layer thickness is less than $100 \mathrm{~nm}$ which is sufficient for efficient evanescent coupling between the III-V layers and the silicon waveguides. The process shows good robustness 
and solid bonding strength and we believe it can provide a promising alternative to direct bonding technique for the fabrication of evanescently-coupled photonic devices. The process can be scaled-up to a multiple die-to-wafer bonding procedure, which would bring the fabrication process closer to an industrial-scale level.

\section{Acknowledgments}

This work was supported by a grant from Intel Corporation. 


\section{References}

1. D. Van Thourhout, G. Roelkens, R. Baets, W. Bogaerts, J. Brouckaert, P. Debackere, P. Dumon, S. Scheerlinck, J. Schrauwen, D. Taillaert, F. Van Laere and J. Van Campenhout, Semicond. Sci. Technol., 23, 064004, (2008).

2. A.W. Fang, H. Park, R. Jones, O. Cohen, M. J. Paniccia, and J. E. Bowers, IEEE Photonic Tech. L., 18, 1143, (2006).

3. J. Van Campenhout, P. Rojo-Romeo, P. Regreny, C. Seassal, D. Van Thourhout, S. Verstuyft, L. Di Cioccio, J.-M. Fedeli, C. Lagahe, and R. Baets, Opt. Express, 15, 6744, (2007).

4. H.H. Chang, A.W. Fang, M. N. Sysak, H. Park, R. Jones, O. Cohen, O. Raday, M. J. Paniccia, and J. E. Bowers et al., Opt. Express, 15, 11466, (2007).

5. A.W. Fang, E. Lively, H. Kuo, D. Liang, and J. E. Bowers, Opt. Express, 16, 4413, (2008).

6. S. Matsuo, T. Nakahara, K. Tateno, and T. Kurokawa, IEEE Photonic Tech. L., 8, 1507, (1996).

7. F. J. Blanco, M. Agirregabiria, J. Garcia, J. Berganzo, M. Tijero, M. T. Arroyo, J. M. Ruano, I. Aramburu, and K. Mayora, J. Micromech. Microeng., 14, 1047 (2004).

8. F. Niklaus, P. Enoksson, E. Kalvesten, and G. Stemme, J. Micromech. Microeng., 11, 100 (2001).

9. G. Roelkens, J. Brouckaert, D. Van Thourhout, R. Baets, R. Nötzel, and M. Smit, J. Electrochem. Soc., 153, G1015 (2006). 
10. J. Brouckaert, G. Roelkens, D. Van Thourhout, and R. Baets, J. Lightwave Technol., 25, 1053, (2007).

11. G. Roelkens, D. Van Thourhout, R. Baets, R. Nötzel, and M. Smit, Opt. Express, 14, 8154, (2006).

12. L. Liu, G. Roelkens, J. Van Campenhout, J. Brouckaert, D. Van Thourhout, and R. Baets, J. Nanosci. Nanotechno., 10, 1461, (2010).

13. http://www.dow.com/cyclotene/prod/302235.htm (Processing Procedures for CYCLOTENE 3000 Series Dry Etch Resins)

14. S. Stankovic, G. Roelkens, D. Van Thourhout, R. Baets, R. Jones, M. Sysak and B. Koch, Proc. 13th Annu. Symp. IEEE/LEOS Benelux Chapter, Netherlands, p.139-142, (2008).

15. F. Zimmer, F. Niklaus, M. Lapisa, T. Ludewig, M. Bring, M. Friedrichs, T. Bakke, H. Schenk and W. van der Wijngaart, Proc. SPIE-Int. Soc. Opt. Eng., 7208, 720807-1, (2009). 


\section{Figures}

Figure 1.

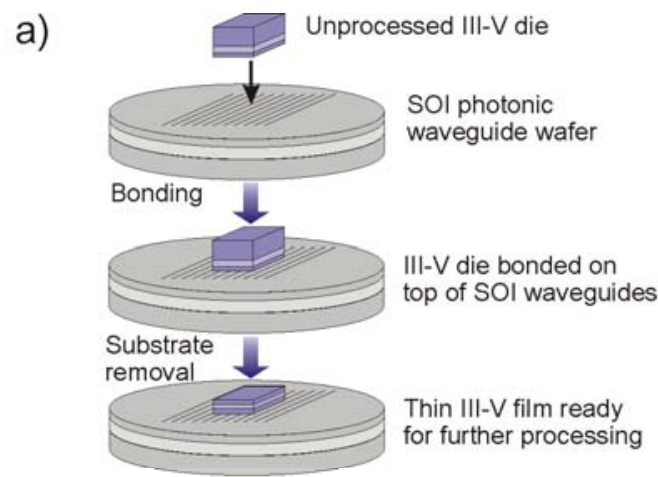

b)


Figure 2.

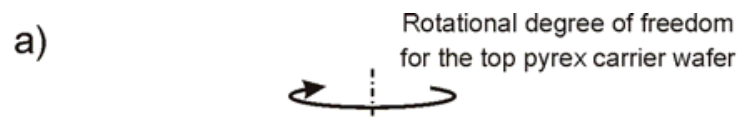

\begin{tabular}{|c|c|c|}
\hline Spacer & Top Pyrex glassicarrier wafer \\
\hline Bottom Pyrex glassicarrier wafer & Spacer \\
\hline Metallic spacers are present
\end{tabular}

b)

\begin{tabular}{|c|}
\hline Top Pyrex glass carrier wafer \\
\hline Bottom Pyrex glass carrier wafer \\
Metallic spacers are removed \\
\hline
\end{tabular}


Figure 3.

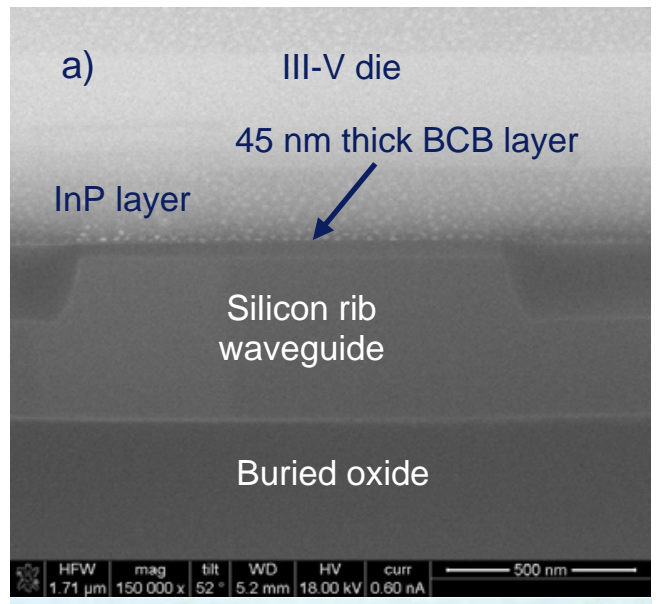

b)

III-V thin film bonded on

top of the waveguides

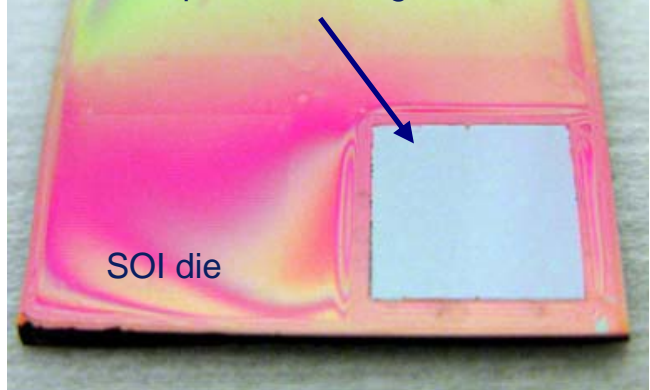


Figure 4.

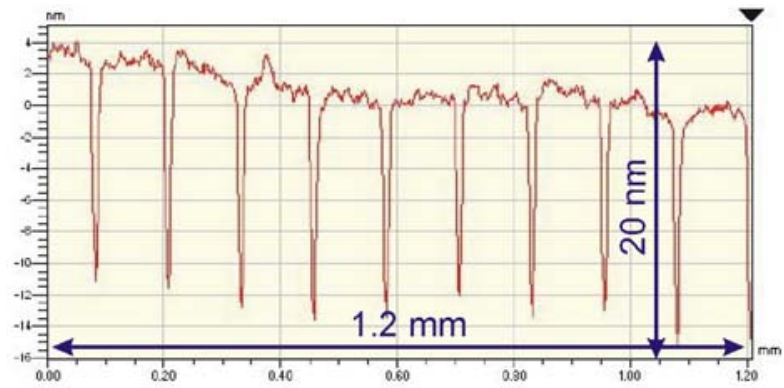




\section{Figure Captions}

Figure 1. a) Heterogeneous integration of a III-V die on top of SOI waveguides based on bonding; b) III-V die comprising InGaAlAs active layers bonded on top of a silicon rib waveguide structure.

Figure 2. Die-to-die bonding setup cross-section: a) prior to bonding - the spacers are in place; b) after the spacers are retracted - III-V and SOI dies come into contact.

Figure 3. a) SEM of the 45nm-thick BCB bonding layer on top of the silicon waveguide, obtained using 1:3 BCB:mesitylene solution; b) III-V film bonded on SOI die after the wet etching: the use of adhesion promoter AP3000 improved the bonding yield. Nonuniformity seen around the III-V film is due to a protective layer of the photoresist.

Figure 4. BCB surface profile perpendicular to SOI waveguides. Notches $(<20 \mathrm{~nm}$ deep $)$ are visible at the locations of the trenches, surrounding the silicon rib waveguides (as shown in Figure 1b), at $125 \mu \mathrm{m}$ pitch. 